\title{
Analysis of Unsteady MHD Thin Layer Flow of Fourth-Order Fluid Through a Vertical Belt
}

\author{
Mohammad Osama Zaheer*, Asif Ali Shaikh and Fozia Shaikh \\ Mehran University of Engineering and Technology, Jamshoro 76090, Pakistan; Osama. \\ mth.st@gmail.com, asif.shaikh@faculty.muet.edu.pk, fozia.shaikh@faculty.muet.edu.pk
}

\begin{abstract}
Article Type: Article
Article Citation: Mohammad Osama Zaheer, Asif Ali Shaikh, Fozia Shaikh. Analysis of unsteady MHD thin layer flow of fourth-order fluid through a vertical belt. Indian Journal of Science and Technology. 2020; 13(03), 299-315. DOl: 10.17485/ijst/2020/ v013i01/148554
\end{abstract}

Received date: 0 ctober 31, 2019

Accepted date: November 30, 2019

*Author for correspondence: Mohammad Osama Zaheer Osama.mth.st@gmail.com P Mehran University of Engineering and Technology, Jamshoro 76090, Pakistan

\begin{abstract}
Objectives: To study the unsteady magneto-hydro-dynamic (MHD) thin layer flow of a fourth-order fluid through a moving and oscillating belt. Methods: The well-known analytical technique, namely Adomian decomposition method (ADM) is used to solve the non-linear partial differential equation for governing equations of velocity profile with subjects to initial and boundary conditions for both lift and drainage problems. Findings and novelty/ improvements: The solution is found in excellent agreement. The basic purpose to study the effect of MHD on velocity field and understand the behavior of this physical problem and the effects of different non-dimensional parameters the graphical results are provided.
\end{abstract}

Keywords: Unsteady Flow, Thin Films/Layers, Fourth-Order Fluid, Magneto-hydro-Dynamics, Non-Newtonian Fluid, Adomian Decomposition Method.

\section{Introduction}

In researched articles, the various structures of non-Newtonian fluids have been advised to modify the research because of the heterogeneous physical nature of these fluids. These fluids are divided into three different representative types of non-Newtonian fluids (a) differential (b) the rate and (c) the integral types fluid models. But, the (a) and (b) types are the most generous and applicable. Therefore, we will study type (a) model in my research i.e. the differential type fluids model. Furthermore, these types are categorized into different sub-classes, it follows (a) the second-order fluid (b) the third-order fluid (c) the fourth-order fluid, etc. The easiest subtype is the second-order fluids also known as grade 2 fluid by which one can sensibly has an expectation to investigate the solution by analytical technique for the predictions of normal stress differences. But it drew back to consider the shearing thickness and shearing thinness cases that exclude the many fluids. On the contrary, the (b) subtype is also known as grade 3 fluid shows an un-idealistic descriptive behavior of these types of fluids. This fluid model can abduct the non-Newtonian behavior 
for instance normal stresses and shearing thickness or shearing thinness. Moreover, the another sub-model of differential type consider as a fourth-order fluid classification or model which captures the greatest amount of fluids flow at one time. In Ref. [1], Gul et al. investigated the velocity profile of fourth order unsteady thin film fluid flow on a vibrating and vertical moving belt.

They applied the Adomian decomposition method (ADM) and optimal homotopy asymptotic method (OHAM) to solve the non-linear differential model and also compared the results of these two methods. In Ref. [2], Gul et al. analyzed the transferring of heat with magneto-hydro-dynamic (MHD) second grade unsteady flow of thin films on a vertical belt. They solved the non-linear differential equation by ADM and OHAM techniques. In Ref. [3], Gul et al. studied the third grade unsteady flow of thin films with MHD in a medium of porosity on an inclined belt with oscillating movement. The nonlinear differential model has been solved by well-known analytical methods homotopy perturbation method (HPM) and OHAM. In Ref. [5], authors examined the heat flowing and transferring of mass with Hydrodynamic stability and fourth-order radiative fluid past a vertically porous plate which reacts chemically. In Ref. [6], Akinshilo analyzed the transferring of heat of third-order non-Newtonian fluid over a porous medium and conveyed the heat internally by parallel plates. In Ref. [7], the unsteady MHD with fourth grade fluid flow past a porous plate has been examined. In Ref. [8], heat transfer analysis of MHD on a vertical belt of a third grade fluid flow of thin films with slipping conditions. $\mathrm{ADM}$ and OHAM have been applied to solve the differential model.

The main objective of this article is to analyze the unsteady MHD thin film flow of nonNewtonian fluid through a vertical oscillating and moving belt using ADM. In Ref. [9], Gul et al. investigated the flow of third-order thin films fluid model over a belt which is vertical with a viscosity that depends on temperature of MHD. They solved the differential model by well-known analytical methods ADM and OHAM. In Ref. [10], Aiyesimi et al. analyzed the transferring of heat of unsteady MHD third grade fluid flow of thin films in the absence of slipping conditions on boundary down an inclined plane. In Ref. [11], Siddiqui et al. analyzed the flow of thin films for a third-order fluid on a moving belt the velocity profile and volume flux was computed by traditional perturbation technique and HPM. In Ref. [12], Zaman et al. studied the effect of hall current of Couette flow for unsteady MHD fourth-order fluid by applying the pressure homotopy technique is used to solve the modeled differential equation.

\section{Basic Equations}

The basic equations which govern the problems are continuity and momentum equations with the interference of transversely magnetic field which is applied externally are as follows,

$$
\begin{gathered}
\nabla \cdot V=0 \\
\rho \frac{D V}{D t}=\nabla \cdot T+\rho g+J \times B
\end{gathered}
$$


Here $\rho$ is the density constant, $g$ represents the gravity, $V$ denotes the velocity vector of fluid, $\frac{D}{D t}$ is material derivative, and $T$ is a cauchy stress tensor, and the term of body force corresponds to MHD flow is the Lorentz force $J \times B$, where $B$ is applied magnetic field and $J$ shows the density of current, according to ohm's law, density of current is given as,

$$
J=\sigma(E+V+B)
$$

Here $\sigma$ and $E$ are electrical conductivity and electric field of the fluid, the imposed magnetic field is denoted by $B=B_{0}+b_{1}$ and $b_{1}$ denotes the magnetic field which is induced. Here we also assume $E=0, b_{1}=0$ and $B=B_{0}\left(0, B_{0}, 0\right)$, where $B_{0}$ is the magnitude of total magnetic field, then (3) reduces to;

$$
J \times B=\left(0, \sigma B_{0}^{2} v(x, t), 0\right)
$$

The Cauchy stress tensor $\mathbf{T}$ which is define for fourth-order fluid as,

$$
T=-p \mathrm{I}+\mu A_{1}+\sum_{r=1}^{3} S_{r}
$$

Here $p$ is pressure, I is an identity tensor while $S_{r}$ denotes the extra stress tensor define as,

$$
\begin{aligned}
S_{1}= & \alpha_{1} A_{2}+\alpha_{2} A_{1}^{2}, S_{2}=\beta_{1} A_{3}+\beta_{2}\left(A_{1} A_{2}+A_{2} A_{1}\right)+\beta_{3}\left(\operatorname{tr} A_{1}^{2}\right) A_{1}, \\
S_{3}= & \gamma_{1} A_{4}+\gamma_{2}\left(A_{3} A_{1}+A_{1} A_{3}\right)+\gamma_{3} A_{2}^{2}+\gamma_{4}\left(A_{2} A_{1}^{2}+A_{1}^{2} A_{2}\right) \\
& +\gamma_{5}\left(t r A_{2}\right) A_{2}+\gamma_{6}\left(t r A_{2}\right) A_{1}^{2}+\left\{\gamma_{7}\left(\operatorname{tr} A_{3}\right)+\gamma_{8}\left(\operatorname{tr} A_{2} A_{1}\right)\right\} A_{1}
\end{aligned}
$$

Where $\mu$ shows the viscosity's coefficient,

$\left(\alpha_{1}, \alpha_{2}\right)\left(\beta_{1}, \beta_{2}, \beta_{3}\right)$ and $\left(\gamma_{1}, \gamma_{2}, \gamma_{3}, \gamma_{4}, \gamma_{5}, \gamma_{6}, \gamma_{7}, \gamma_{8}\right)$ are the material constants of the second, third, and fourth-order of fluids. Furthermore, the Rivlin-Ericksen tensor $A_{1}, A_{2}$, $A_{3}$, and $A_{4}$ are defined as;

$$
\begin{gathered}
A_{0}=\mathrm{I} \\
A_{1}=(\nabla V)+(\nabla V)^{T}, \\
A_{k}=\frac{D A_{k-1}}{D t}+A_{k-1}(\nabla V)+(\nabla V)^{T} A_{k-1}, k=2,3,4
\end{gathered}
$$

\section{Formulation of Lift Problem}

We consider two different problems of a wide vertical belt with uniform thickness $\delta$ of a thin film layer of fourth-order fluid placed in a large bath. On the belt the transversely uniform magnetic field is applied. For analysis, we have chosen the Cartesian coordinate 
system and flow in assumed to be one dimensional in the direction of $y$ where as $x$ is normal to it, we suppose that the flow is unsteady and laminar with no pressure gradient the gravity is kept balanced by shearing forces of fluid and the thickness of the films does not change and by the above conditions the velocity is in $y$-direction [1]:

$$
V=(0, v(x, t), 0)
$$

Here we consider the flow at $t=0^{+}$on a wide belt which is oscillating and moving upward with the velocity $U$. The belt is dipped into a bath as shown in

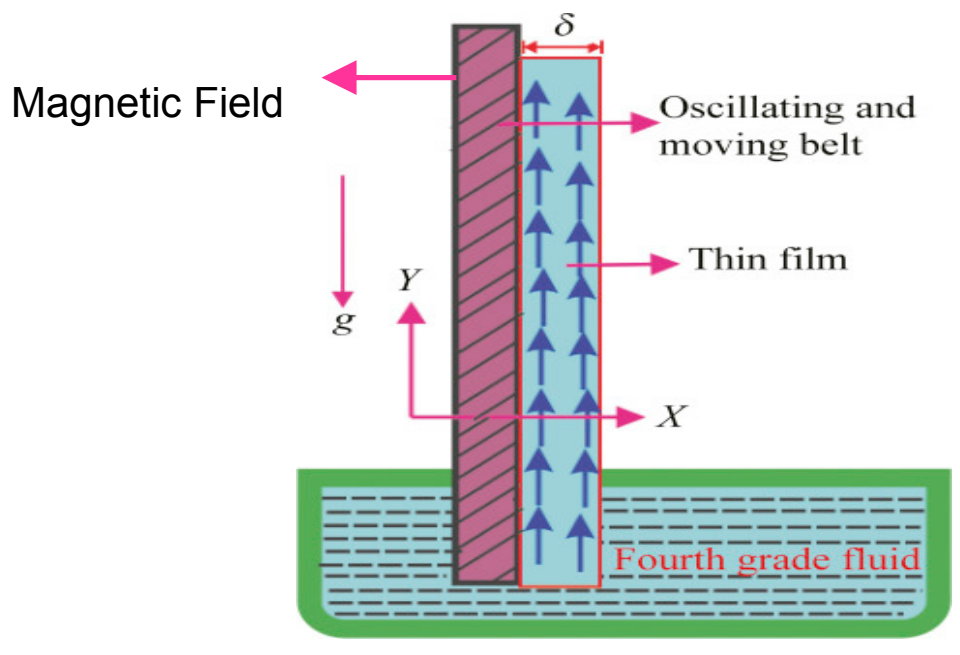

FIGURE 1. Geometry of lift problem.

With the boundary conditions

$$
\begin{gathered}
v(x, t)=U+U \cos \omega t, \text { at } x=0, \\
\frac{\partial v(x, t)}{\partial x}=0, \text { at } x=\delta
\end{gathered}
$$

Where the belt which is oscillating whose frequency is $\omega$.

Using the velocity profile given in equation (10), the continuity equation (1) is identically satisfied and the equation of momentum (2) becomes to the form

$$
\rho \frac{\partial v}{\partial t}=\frac{\partial}{\partial x} T_{x y}-\rho g-\sigma B_{o}^{2} v
$$

From Equation (5), the component $T_{x y}$ of Cauchy stress component is

$$
\begin{aligned}
T_{x y}= & \mu \frac{\partial v}{\partial x}+\alpha_{1} \frac{\partial}{\partial t}\left(\frac{\partial v}{\partial x}\right)+\beta_{1} \frac{\partial^{2}}{\partial t^{2}}\left(\frac{\partial v}{\partial x}\right)+2\left(\beta_{2}+\beta_{3}\right)\left(\frac{\partial v}{\partial x}\right)^{3} \\
& +\gamma_{1} \frac{\partial^{3}}{\partial t^{3}}\left(\frac{\partial v}{\partial x}\right)+2\left(3 \gamma_{2}+\gamma_{3}+\gamma_{4}+\gamma_{5}+3 \gamma_{7}+\gamma_{8}\right) \frac{\partial}{\partial t}\left(\frac{\partial v}{\partial x}\right)^{3}
\end{aligned}
$$


Inserting Equation (14) into Equation (13) we get

$$
\begin{aligned}
& \frac{\partial v}{\partial t}=\mu \frac{\partial^{2} v}{\partial x^{2}}+\alpha_{1} \frac{\partial}{\partial t}\left(\frac{\partial^{2} v}{\partial x^{2}}\right)+\beta_{1} \frac{\partial^{2}}{\partial t^{2}}\left(\frac{\partial v}{\partial x}\right)+6\left(\beta_{2}+\beta_{3}\right)\left(\frac{\partial v}{\partial x}\right)^{2}\left(\frac{\partial^{2} v}{\partial x^{2}}\right) \\
& +\gamma_{1} \frac{\partial^{3}}{\partial t^{3}}\left(\frac{\partial^{2} v}{\partial x^{2}}\right)+6\left(3 \gamma_{2}+\gamma_{3}+\gamma_{4}+\gamma_{5}+3 \gamma_{7}+\gamma_{8}\right) \frac{\partial}{\partial t}\left(\frac{\partial v}{\partial x}\right)^{2} \frac{\partial^{2} v}{\partial x^{2}}-\rho g-\sigma B_{o}^{2} v
\end{aligned}
$$

Introducing the following non-dimensional variables

$$
\begin{gathered}
\tilde{v}=\frac{v}{U}, \tilde{x}=\frac{x}{\delta}, \tilde{t}=\frac{u t}{\rho \delta^{2}}, S_{t}=\frac{\delta^{2} \rho g}{k}, \\
\tilde{\alpha}=\frac{\alpha_{1}}{\rho \delta^{2}}, \quad \tilde{\beta}_{1}=\frac{\beta_{1} \mu}{\delta^{2}}, \tilde{\gamma}_{1}=\frac{\gamma_{1} \mu^{2}}{\rho^{3} \delta^{4}}, \\
\tilde{\beta}=\frac{\left(\beta_{2}+\beta_{3}\right) U^{2}}{\mu \delta^{2}}, M=\frac{\sigma B_{o}^{2} \delta^{2}}{\mu_{0}}, \tilde{\gamma}=\left(3 \gamma_{2}+\gamma_{3}+\gamma_{4}+\gamma_{5}+3 \gamma_{7}+\gamma_{8}\right) \frac{\gamma_{1} U^{2}}{\rho \delta^{4}}
\end{gathered}
$$

Here $\tilde{t}$ is a non-dimensional time variable, $\tilde{\alpha}$ is non-dimensional second-order nonNewtonian variable, $\tilde{\beta}, \tilde{\beta}_{1}$ are non-dimensional third-order non-Newtonian variable and $\tilde{\gamma}, \tilde{\gamma}_{1}$ are non-dimensional fourth grade non-Newtonian variable, is a stock number, and $\mathrm{M}$ is a non-dimensional magnetic variable.

On inserting these non-dimensionless parameters in (15) and boundary conditions (11) and (12), we get.

$$
\begin{gathered}
\frac{\partial v}{\partial t}=\mu \frac{\partial^{2} v}{\partial x^{2}}+\alpha_{1} \frac{\partial}{\partial t}\left(\frac{\partial^{2} v}{\partial x^{2}}\right)+\beta_{1} \frac{\partial^{2}}{\partial t^{2}}\left(\frac{\partial v}{\partial x}\right)+6\left(\beta_{2}+\beta_{3}\right)\left(\frac{\partial v}{\partial x}\right)^{2}\left(\frac{\partial^{2} v}{\partial x^{2}}\right) \\
+\gamma_{1} \frac{\partial^{3}}{\partial t^{3}}\left(\frac{\partial^{2} v}{\partial x^{2}}\right)+6\left(3 \gamma_{2}+\gamma_{3}+\gamma_{4}+\gamma_{5}+3 \gamma_{7}+\gamma_{8}\right) \frac{\partial}{\partial t}\left(\frac{\partial v}{\partial x}\right)^{2} \frac{\partial^{2} v}{\partial x^{2}}-\rho g-\sigma B_{o}^{2} v \\
v(x, t)=1+\cos \omega t, \text { at } x=0, \\
\frac{\partial v(x, t)}{\partial x}=0, \text { at } x=1,
\end{gathered}
$$

\section{Formulation of Drainage Problem}

Here we assume the belt is oscillating but not moving and the fluid falls downward direction in the presence of gravity, as shown in the geometry of drainage problem See Figure 


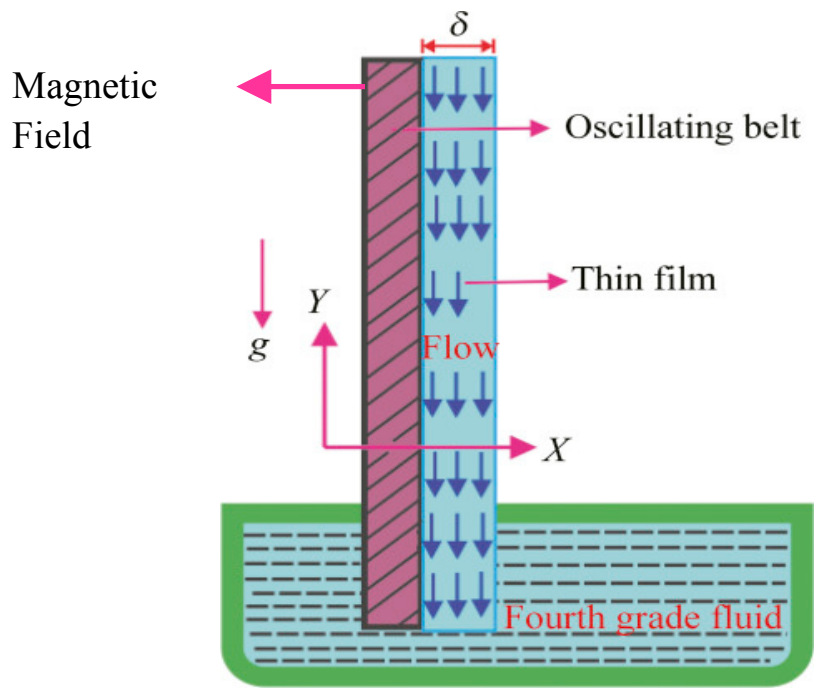

FIGURE 2. Geometry of drainage problem.

Along with the boundary conditions

$$
\begin{gathered}
v(x, t)=U \cos \omega t, \text { at } x=0, \\
\frac{\partial v(x, t)}{\partial x}=0, \text { at } x=\delta,
\end{gathered}
$$

All the remaining assumptions are similar to the lift problem but stock number is taken positive. Using $T_{x y}$ the governing equation of drainage problem.

$$
\begin{aligned}
\rho \frac{\partial v}{\partial t}= & \mu \frac{\partial^{2} v}{\partial x^{2}}+\alpha_{1} \frac{\partial}{\partial t}\left(\frac{\partial^{2} v}{\partial x^{2}}\right)+\beta_{1} \frac{\partial^{2}}{\partial t^{2}}\left(\frac{\partial v}{\partial x}\right)+6\left(\beta_{2}+\beta_{3}\right)\left(\frac{\partial v}{\partial x}\right)^{2}\left(\frac{\partial^{2} v}{\partial x^{2}}\right)+\gamma_{1} \frac{\partial^{3}}{\partial t^{3}}\left(\frac{\partial^{2} v}{\partial x^{2}}\right) \\
& +6\left(3 \gamma_{2}+\gamma_{3}+\gamma_{4}+\gamma_{5}+3 \gamma_{7}+\gamma_{8}\right) \frac{\partial}{\partial t}\left(\frac{\partial v}{\partial x}\right)^{2} \frac{\partial^{2} v}{\partial x^{2}}-\rho g-\sigma B_{o}^{2} v
\end{aligned}
$$

The non-dimensional form of equation (22) is given by,

$$
\begin{gathered}
\frac{\partial v}{\partial t}=S_{t}+\frac{\partial^{2} v}{\partial x^{2}}+\alpha \frac{\partial}{\partial t}\left(\frac{\partial^{2} v}{\partial x^{2}}\right)+\beta_{1} \frac{\partial^{2}}{\partial t^{2}}\left(\frac{\partial v}{\partial x}\right)+6 \beta\left(\frac{\partial v}{\partial x}\right)^{2}\left(\frac{\partial^{2} v}{\partial x^{2}}\right) \\
+\gamma_{1} \frac{\partial^{3}}{\partial t^{3}}\left(\frac{\partial^{2} v}{\partial x^{2}}\right)+6 \gamma \frac{\partial}{\partial t}\left(\frac{\partial v}{\partial x}\right)^{2} \frac{\partial^{2} v}{\partial x^{2}}-\rho g-\sigma B_{o}^{2} v \\
v(x, t)=\cos \omega t, \text { at } x=0, \\
\frac{\partial v(x, t)}{\partial x}=0, \text { at } x=1,
\end{gathered}
$$




\section{Solution Technique}

Fundamental concept of ADM

$\mathrm{ADM}$ is used to decompose the unknown function $u(x, t)$ into a sum of an infinite number of components defined the decomposition series.

$$
u(x, t)=\sum_{n=0}^{\infty} u_{n}(x, t)
$$

The decomposition method is used to find the components

$$
u_{0}(x, t), u_{1}(x, t), u_{2}(x, t) \ldots
$$

separately. The Determination of these components can be obtained through simple integrals. To give a clear overview of $\mathrm{ADM}$, we consider the partial differential equation in an operator form as

$$
\begin{aligned}
& L_{t} u(x, t)+L_{x} u(x, t)+R u(x, t)+N u(x, t)=g(x, t) \\
& L_{t} u(x, t)=g(x, t)-L_{x} u(x, t)-R u(x, t)-N u(x, t)
\end{aligned}
$$

where $L_{x}=\frac{\partial^{2}}{\partial x^{2}}$ and $L_{t}=\frac{\partial}{\partial t}$ are used in partial differential equation as linear operators and simply invertible, $g(x, t)$ is a term of source, $R u(x, t)$ is a residual linear term, and $N u(x, t)$ is analytical term which is non-linear and can be expanded in the $A_{n}$ that is $A$ domian polynomials.

After applying the inverse operator $L_{x}^{-1}$ to both sides of Equation (28), we write

$$
\begin{gathered}
L_{x}^{-1} L_{t} u(x, t)=L_{x}^{-1} g(x, t)-L_{x}^{-1} L_{t} u(x, t)-L_{x}^{-1} R u(x, t)-L_{x}^{-1} N u(x, t) \\
u(x, t)=f(x, t)-L_{x}^{-1} L_{t} u(x, t)-L_{x}^{-1} R u(x, t)-L_{x}^{-1} N u(x, t)
\end{gathered}
$$

Here the function $f(x, t)$ shows the terms arising form $L_{x}^{-1} g(x, t)$ after using the condition and the inverse operator $L_{x}^{-1}=\iint{ }^{x}\left({ }^{*}\right) d x d x$ which is used for second-order partial differential equation (P.D.E)

The series of this method $u(x, t)$ is defined as:

$$
\begin{gathered}
u(x, t)=\sum_{n=0}^{\infty} u_{n}(x, t) \\
\sum_{n=0}^{\infty} u_{n}(x, t)=f(x, t)-L_{x}^{-1} L_{t} \sum_{n=0}^{\infty} u(x, t)-L_{x}^{-1} R \sum_{n=0}^{\infty} u(x, t)-L_{x}^{-1} N \sum_{n=0}^{\infty} u(x, t)
\end{gathered}
$$

The term expanded which is non-linear in Adomian decomposition polynomials as: 


$$
N(v(x, t))=\sum_{n=0}^{\infty} A_{n}
$$

Where the components $u_{0}(x, t), u_{1}(x, t), u_{2}(x, t) \ldots$ are periodically derived as:

$$
\begin{aligned}
& u_{0}(x, t)+u_{1}(x, t)+u_{2}(x, t)+\ldots \\
& =f(x, t)-L_{x}^{-1} L_{t}\left(u_{0}(x, t)+u_{1}(x, t)+u_{2}(x, t)+\ldots\right) \\
& -L_{x}^{-1} R\left(u_{0}(x, t)+u_{1}(x, t)+u_{2}(x, t)+\ldots\right) \\
& -L_{x}^{-1} N\left(A_{0}+A_{1}+A_{2}+\ldots\right)
\end{aligned}
$$

To govern the components of the series $u_{0}(x, t)+u_{1}(x, t)+u_{2}(x, t)+\ldots$, it is significant to note that $\mathrm{ADM}$ proposes that the zeroth module of $u_{0}(x, t)$ is usually distinct by the function $f(x, t)$ describes as:

$$
\begin{aligned}
& u_{0}(x, t)=f(x, t), u_{1}(x, t)=-L_{x}^{-1} L_{t}\left(u_{0}(x, t)\right)-L_{x}^{-1} R\left(u_{0}(x, t)\right)-L_{x}^{-1} N\left(A_{0}\right) \\
& u_{2}(x, t)=-L_{x}^{-1} L_{t}\left(u_{1}(x, t)\right)-L_{x}^{-1} R\left(u_{1}(x, t)\right)-L_{x}^{-1} N\left(A_{1}\right) \\
& u_{3}(x, t)=-L_{x}^{-1} L_{t}\left(u_{2}(x, t)\right)-L_{x}^{-1} R\left(u_{2}(x, t)\right)-L_{x}^{-1} N\left(A_{2}\right)
\end{aligned}
$$

And so on.

\section{The ADM Solution of Lift Problem}

Rewrite Equation (17) in the L operator form of $\mathrm{ADM}$, we get

$$
\begin{aligned}
L_{x} v(x, t)= & S_{t}+\frac{\partial v}{\partial t}+M v-\alpha \frac{\partial}{\partial t}\left(\frac{\partial^{2} v}{\partial x^{2}}\right) \\
& -\beta_{1} \frac{\partial^{2}}{\partial t^{2}}\left(\frac{\partial v}{\partial x}\right)-6 \beta\left(\frac{\partial v}{\partial x}\right)^{2}\left(\frac{\partial^{2} v}{\partial x^{2}}\right) \\
& -\gamma_{1} \frac{\partial^{3}}{\partial t^{3}}\left(\frac{\partial^{2} v}{\partial x^{2}}\right)-6 \gamma \frac{\partial}{\partial t}\left(\frac{\partial v}{\partial x}\right)^{2} \frac{\partial^{2} v}{\partial x^{2}}
\end{aligned}
$$

Using the inverse operator $L_{x}^{-1}$, we get

$$
\begin{aligned}
L_{x} v(x, t)=S_{t} & +\frac{\partial v}{\partial t}+M v-\alpha \frac{\partial}{\partial t}\left(\frac{\partial^{2} v}{\partial x^{2}}\right) \\
& -\beta_{1} \frac{\partial^{2}}{\partial t^{2}}\left(\frac{\partial v}{\partial x}\right)-6 \beta\left(\frac{\partial v}{\partial x}\right)^{2}\left(\frac{\partial^{2} v}{\partial x^{2}}\right) \\
& -\gamma_{1} \frac{\partial^{3}}{\partial t^{3}}\left(\frac{\partial^{2} v}{\partial x^{2}}\right)-6 \gamma \frac{\partial}{\partial t}\left(\frac{\partial v}{\partial x}\right)^{2} \frac{\partial^{2} v}{\partial x^{2}}
\end{aligned}
$$




$$
\begin{aligned}
\sum_{n=0}^{\infty} v(x, t)=b_{0} & +b_{1} x+S_{t} \frac{x^{2}}{2}+M L_{x}^{-1}\left(\sum_{n=0}^{\infty} v_{n}\right) \\
& -\alpha L_{x}^{-1}\left[\sum_{n=0}^{\infty} A_{n}\right]-\beta_{1} L_{x}^{-1}\left[\sum_{n=0}^{\infty} B_{n}\right]-6 \beta L_{x}^{-1}\left[\sum_{n=0}^{\infty} C_{n}\right] \\
& -\gamma_{1} L_{x}^{-1}\left[\sum_{n=0}^{\infty} D_{n}\right]-6 \gamma L_{x}^{-1}\left[\sum_{n=0}^{\infty} E_{n}\right]
\end{aligned}
$$

ere $A_{n}, B_{n}, C_{n}, D_{n}$, and $E_{n}$ are adomian polynomials and define as,

$$
\begin{gathered}
\sum_{n=0}^{\infty} A_{n}=\frac{\partial}{\partial t}\left(\frac{\partial^{2} v}{\partial x^{2}}\right) ; \sum_{n=0}^{\infty} B_{n}=\frac{\partial^{2}}{\partial t^{2}}\left(\frac{\partial v}{\partial x}\right) ; \sum_{n=0}^{\infty} C_{n}=\left(\frac{\partial v}{\partial x}\right)^{2}\left(\frac{\partial^{2} v}{\partial x^{2}}\right) \\
\sum_{n=0}^{\infty} D_{n}=\frac{\partial^{3}}{\partial t^{3}}\left(\frac{\partial^{2} v}{\partial x^{2}}\right) ; \sum_{n=0}^{\infty} E_{n}=\frac{\partial}{\partial t}\left(\frac{\partial v}{\partial x}\right)^{2} \frac{\partial^{2} v}{\partial x^{2}} \\
v_{0}+v_{1}+v_{2}+\ldots=b_{0}+b_{1} x+S_{t} \frac{x^{2}}{2}+M L_{x}^{-1}\left(v_{0}+v_{1}+v_{2}+\ldots\right) \\
-\alpha L_{x}^{-1}\left[A_{0}+A_{1}+A_{2}+\ldots\right]-\beta_{1} L_{x}^{-1}\left[B_{0}+B_{1}+B_{2}+\ldots\right]\left[B_{0}+B_{1}+B_{2}+\ldots\right] \\
-6 \beta L_{x}^{-1}\left[C_{0}+C_{1}+C_{2}+\ldots\right]-\gamma_{1} L_{x}^{-1}\left[D_{0}+D_{1}+D_{2}+\ldots\right]-6 \gamma L_{x}^{-1}\left[E_{0}+E_{1}+E_{2}+\ldots\right]
\end{gathered}
$$

The components of velocity profile are obtained by comparing both side of equation (40)

Zeroth component problem:

$$
v_{0}(x, t)=b_{0}+b_{1} x+S_{t} \frac{x^{2}}{2}
$$

Solution of zeroth component problem using boundary conditions given in equation (18) and (19) is:

$$
v_{0}(x, t)=1+\cos (\omega t)-\left(1+\cos (\omega t)+\frac{S_{t}}{2}\right) x+\left(\frac{S_{t}}{2}\right) x^{2}
$$

First component problem:

$$
\begin{aligned}
v_{1}(x, t)= & S_{t}+L_{x}^{-1}\left(\frac{\partial v_{o}}{\partial t}\right)+M L_{x}^{-1}\left(v_{0}\right) \\
& -\alpha L_{x}^{-1}\left[A_{0}\right]-\beta_{1} L_{x}^{-1}\left[B_{0}\right]-6 \beta L_{x}^{-1}\left[C_{0}\right] \\
& -\gamma_{1} L_{x}^{-1}\left[D_{0}\right]-6 \gamma L_{x}^{-1}\left[E_{0}\right]
\end{aligned}
$$


The first component problem solution using boundary conditions given in Equations (18) and (19)

$$
\begin{aligned}
v_{1}(x, t)=v_{1} & (x, t)=\left[\frac{\omega}{3} \sin (\omega t)-\frac{M}{3}-\frac{M}{3} \cos (\omega t)+\frac{M}{24} S_{t}+\frac{\beta}{4} S_{t}^{3}+\beta S_{t}^{2}+\beta S_{t}^{2} \cos (\omega t)-\omega \gamma S_{t}^{2} \sin (\omega t)\right. \\
& \left.+3 \beta S_{t} \cos ^{2}(\omega t)+6 \beta S_{t} \cos (\omega t)+3 \beta S_{t}-6 \omega \gamma S_{t} \cos (\omega t) \cdot \sin (\omega t)-6 \omega \gamma S_{t} \cos (\omega t)\right] \\
& +\left[-\frac{\omega}{2} \sin (\omega t)+\frac{M}{2}+\frac{M}{3} \cos (\omega t)-\frac{3 \beta}{4} S_{t}^{3}-3 \beta S_{t}^{2}+\beta S_{t}^{2} \cos (\omega t)+3 \omega \gamma S_{t}^{2} \sin (\omega t)\right. \\
& \left.-3 \beta S_{t} \cos ^{2}(\omega t)-6 \beta S_{t} \cos (\omega t)-3 \beta S_{t}+6 \omega \gamma S_{t} \cos (\omega t) \cdot \sin (\omega t)\right] x^{2} \\
& +\left[\frac{\omega}{6} \sin (\omega t)-\frac{M}{6}-\frac{M}{6} \cos (\omega t)-\frac{M}{12} S_{t}+\beta S_{t}^{3}+2 \beta S_{t}^{2} \cos (\omega t)-2 \omega \gamma S_{t}^{2} \sin (\omega t)\right] x^{3} \\
& +\left[\frac{M}{24} S_{t}-\frac{\beta}{2} S_{t}^{3}\right] x^{4}
\end{aligned}
$$

Second component problem:

$$
\begin{aligned}
v_{2}(x, t)= & L_{x}^{-1}\left(\frac{\partial v_{1}}{\partial t}\right)+M L_{x}^{-1}\left(v_{1}\right)-\alpha L_{x}^{-1}\left[A_{1}\right] \\
& -\beta_{1} L_{x}^{-1}\left[B_{1}\right]-6 \beta L_{x}^{-1}\left[C_{1}\right]-\gamma_{1} L_{x}^{-1}\left[D_{1}\right] \\
& -6 \gamma L_{x}^{-1}\left[E_{1}\right]
\end{aligned}
$$

The first-order analytical result has been computed while graphical solutions are provided up to second order due to massive calculation (Error! Reference source not found.).

\section{The ADM Solution of Drainage Problem}

The model for drainage problem is the same as for the lift problem. The only difference in this problem is that the belt is only oscillating and due to the draining of thin film, stock number is positively mentioned in Equation (23). After rewriting equation (23) in L operator form of ADM, then using boundary conditions Equations (24) (25) we get the components of the problem are

Zeroth component problem:

$$
v_{0}(x, t)=b_{0}+b_{1} x-S_{t} \frac{x^{2}}{2}
$$

The zeroth component problem solution using boundary conditions which is given in Equations (24) and (25) is

$$
v_{0}(x, t)=\cos (\omega t)-\left(\cos (\omega t)-\frac{S_{t}}{2}\right) x-\left(\frac{S_{t}}{2}\right) x^{2}
$$


First component problem:

$$
\begin{aligned}
v_{1}(x, t)= & -S_{t}+L_{x}^{-1}\left(\frac{\partial v_{o}}{\partial t}\right)+M L_{x}^{-1}\left(v_{0}\right) \\
& -\alpha L_{x}^{-1}\left[A_{0}\right]-\beta_{1} L_{x}^{-1}\left[B_{0}\right]-6 \beta L_{x}^{-1}\left[C_{0}\right] \\
& -\gamma_{1} L_{x}^{-1}\left[D_{0}\right]-6 \gamma L_{x}^{-1}\left[E_{0}\right]
\end{aligned}
$$

The first component problem solution by using boundary conditions which is given in Equations (24) and (25)

$$
\begin{aligned}
v_{1}(x, t)= & {\left[3 \beta S_{t} \cos ^{2}(\omega t)+\frac{\omega}{3} \sin (\omega t)\left(\gamma S_{t}^{2}-1\right)-\omega \gamma S_{t} \sin (2 \omega t)-\beta S_{t}^{2} \cos (\omega t)+\frac{M}{3} S_{t}-\frac{M}{3} \cos (\omega t)+\frac{\beta}{4} S_{t}^{3}\right] x } \\
& +\left[\frac{\omega}{2} \sin (\omega t)\left(1-\gamma S_{t}^{2}\right)-3 \beta S_{t} \cos ^{2}(\omega t)+\omega \gamma S_{t} \sin (2 \omega t)+3 \beta S_{t}^{2} \cos (\omega t)+\frac{M}{2} \cos (\omega t)-\frac{3 \beta}{4} S_{t}^{3}\right] x^{2} \\
& +\left[\frac{\omega}{3} \sin (\omega t)\left(2 \gamma S_{t}^{2}-\frac{1}{2}\right)-2 \beta S_{t}^{2} \cos (\omega t)+\frac{M}{6} S_{t}-\frac{M}{6} \cos (\omega t)+\frac{\beta}{4} S_{t}^{3}\right] x^{3}-\left[\frac{M}{24} S_{t}+\frac{\beta}{2} S_{t}^{3}\right] x^{4}
\end{aligned}
$$

Second component problem:

$$
\begin{aligned}
v_{2}(x, t)= & L_{x}^{-1}\left(\frac{\partial v_{1}}{\partial t}\right)+M L_{x}^{-1}\left(v_{1}\right)-\alpha L_{x}^{-1}\left[A_{1}\right] \\
& -\beta_{1} L_{x}^{-1}\left[B_{1}\right]-6 \beta L_{x}^{-1}\left[C_{1}\right]-\gamma_{1} L_{x}^{-1}\left[D_{1}\right]-6 \gamma L_{x}^{-1}\left[E_{1}\right]
\end{aligned}
$$

The first-order analytical result has been computed while graphical solutions are provided up to second order due to massive calculation (Error! Reference source not found.).

\section{Effects of Non-dimensional Parameters}

\subsection{Results and Discussion}

The analytical solutions are examined for unsteady MHD thin layer flow of fourth-order fluid through a vertical belt which is oscillating and also moving. The arrived non-linear partial differential equations of both drainage and lift problems are solved by using the $\mathrm{ADM}$ and results are also shown by graphically.

Figure show the geometry of lift and drainage problems, respectively. Figure 1 and Figure 2 show the graphical results for the velocity profile of both lift and drainage problems respectively at altered values of the inserted parameters. Whereas the effects of other physical parameters $t, s_{t}, \alpha, \beta, \gamma$ and $M$ on velocity field for both lift and drainage problems are examined in Figures $3-10$. All the results are computed in the $x$-coordinate only for a certain domain $x \in[0,1]$. In Figure 3 and Figure 4 showing the effect of $\beta$ on lift and drainage velocities respectively in both cases we noticed that the velocity profile increases as the value of $\beta$ increases. In general, the Newtonian fluid shows much thinner boundary film than non-Newtonian fluid due to the reduced apparent viscosity. The 


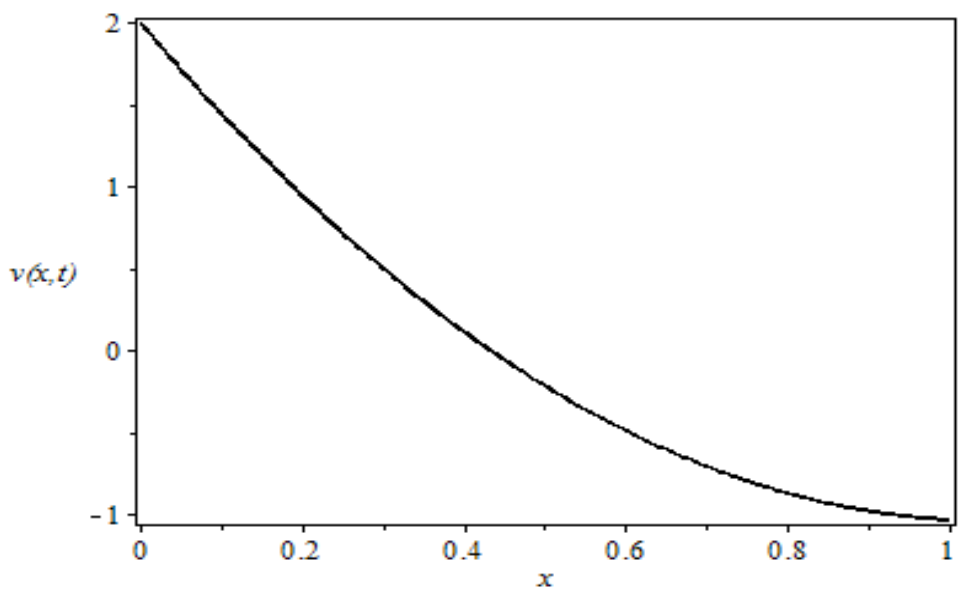

FIGURE 3. ADM technique for velocity profile of lift problem by taking . $\omega=5, \alpha=0.02, \beta$ $=0.4, \gamma=2.0, \beta_{1}=0.1, S_{t}=0.2, \gamma_{1}=0.01, t=3, M=0.5$.

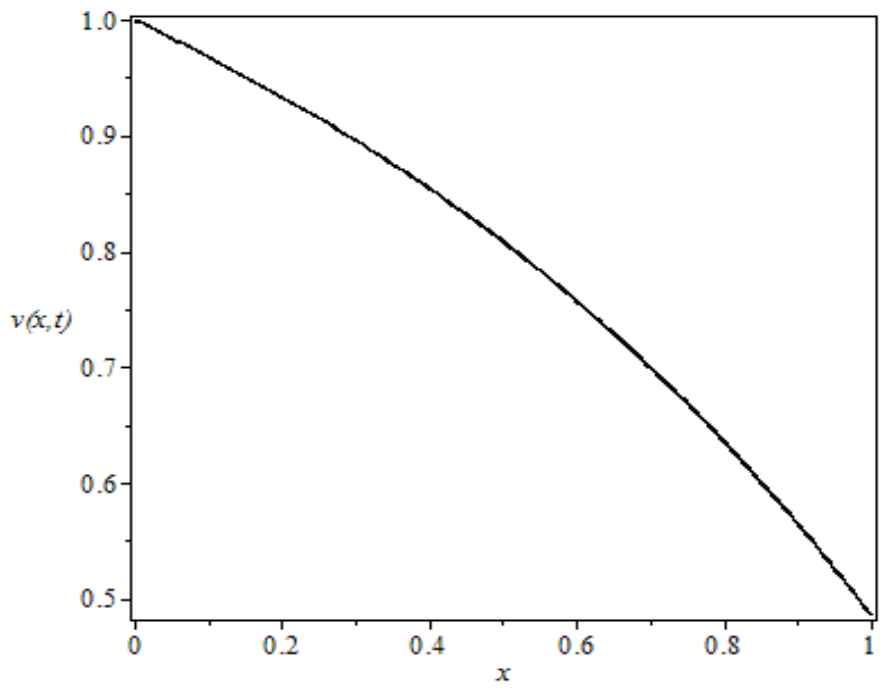

FIGURE 4. ADM technique for drainage velocity profile by using $\omega=5, \alpha=0.02, \beta=0.4$, $\gamma=2.0, \beta_{1}=0.3, S_{t}=0.2, \gamma_{1}=0.04, t=3, M=0.5$.

outward viscosity of the fluid become more bulky (domination of viscous forces) due to the increment in fourth-order parameters then the flow will adjust simultaneously to the driving force which is present and closely oscillate with the congruent phase in the whole domain of flow. Therefore, increment in non-Newtonian parameters $\alpha, \beta, \gamma, \beta_{1}$, and $\gamma_{1}$, of second, third, and fourth-order fluids causes more thickening of the boundary layer. So increment in these parameters increases the field of velocity for both lift and drainage problems shown in Figure 3, Figure 4, Figure 5, Figure 6. The effects of $S_{t}$ are shown in Figure 7 and Figure 8 on lift and drainage velocities, respectively. From Figure 7, we perceived that the lift velocity decreases by increasing the value of $S_{t}$, whereas in Figure 8 


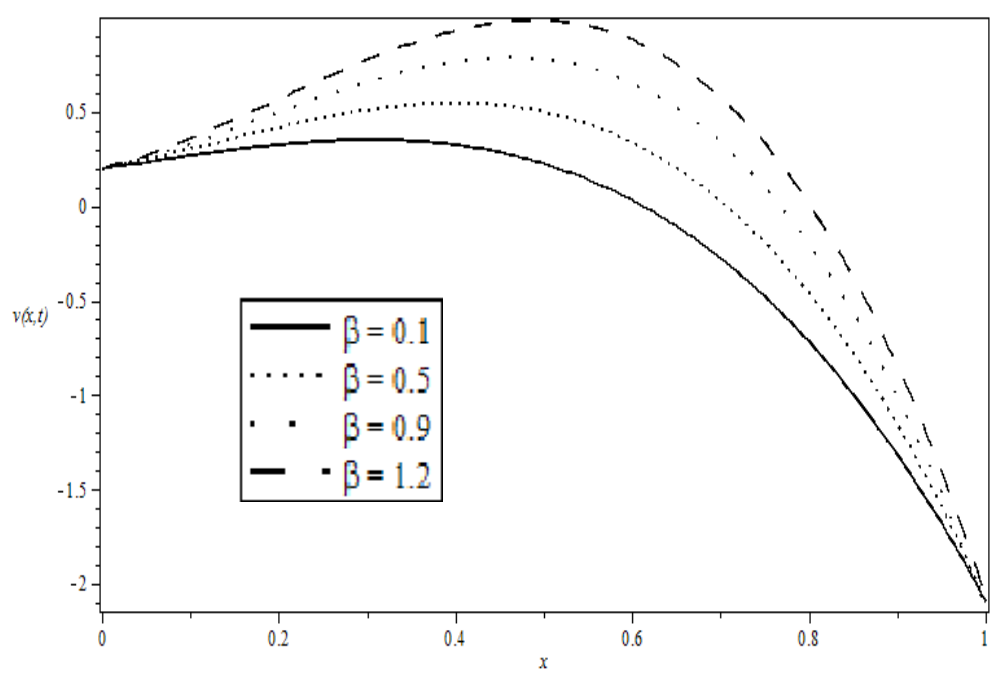

FIGURE 5. Influence of third-order parameter $\beta$ of velocity profile on lift problem when $\alpha=0.2, \omega=5, \gamma=2.0, \beta_{1}=0.7, S_{t}=0.2, \gamma_{1}=0.2, t=3, M=0.5$.

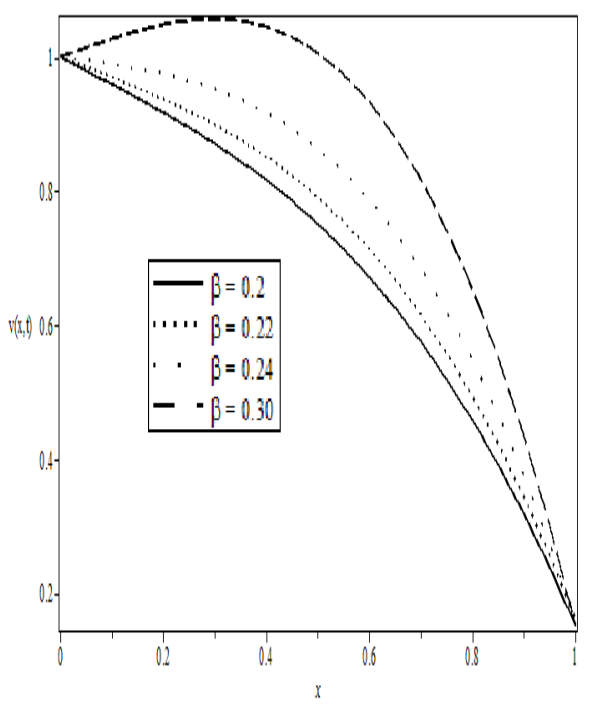

FIGURE 6. Influence of third-order parameter $\beta$ of velocity profile on Drainage problem when $\omega=5, \alpha=0.2, \gamma=2.0, \beta_{1}=0.7, S_{t}=0.2, \gamma_{1}=0.2, t=3, M=0.5$.

increasing in the value of $S_{t}$ drainage velocity increases. This relates to the frictional force that causing the effect of gravity and it appears to be smaller near to the belt. While towards the free surface it gradually increases. In Figure 9 and Figure 10 showing the variation of the magnetic parameter $\mathrm{M}$ on the lift and drainage velocity profiles Increase in magnetic 


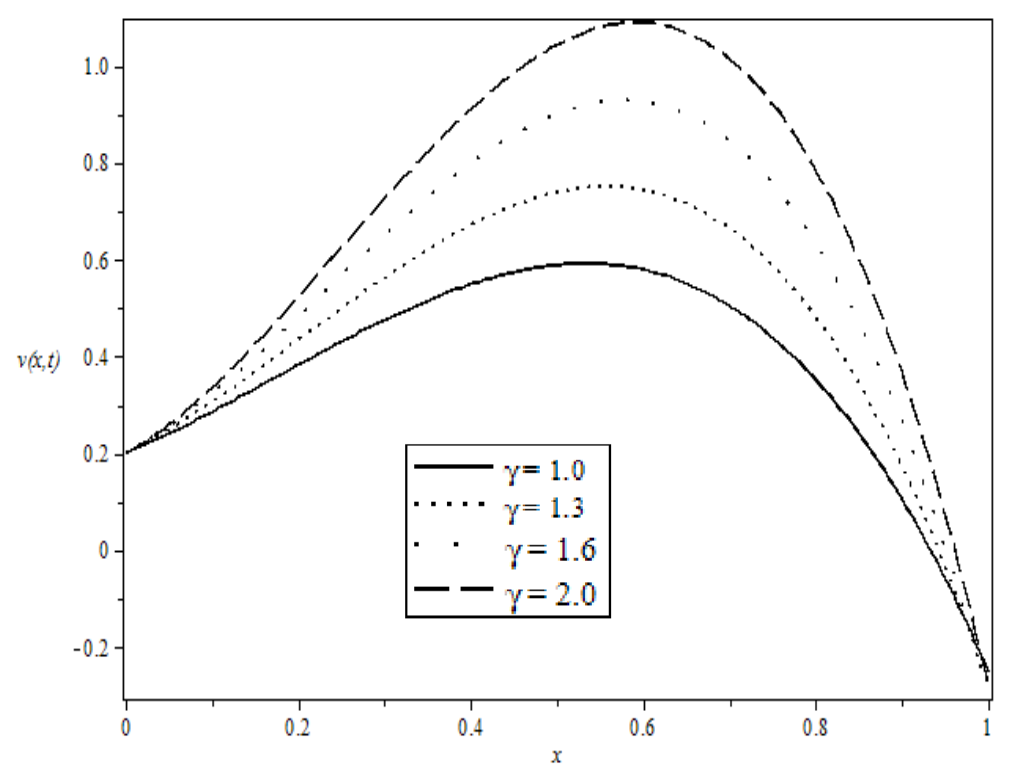

FIGURE 7. Effect of fourth-order parameter $\gamma$ on lift velocity profile when $\omega=5, \alpha=0.2, \beta$ $=0.1, \beta_{1}=0.7, S_{t}=0.2, \gamma_{1}=0.2, t=3, M=0.5$.

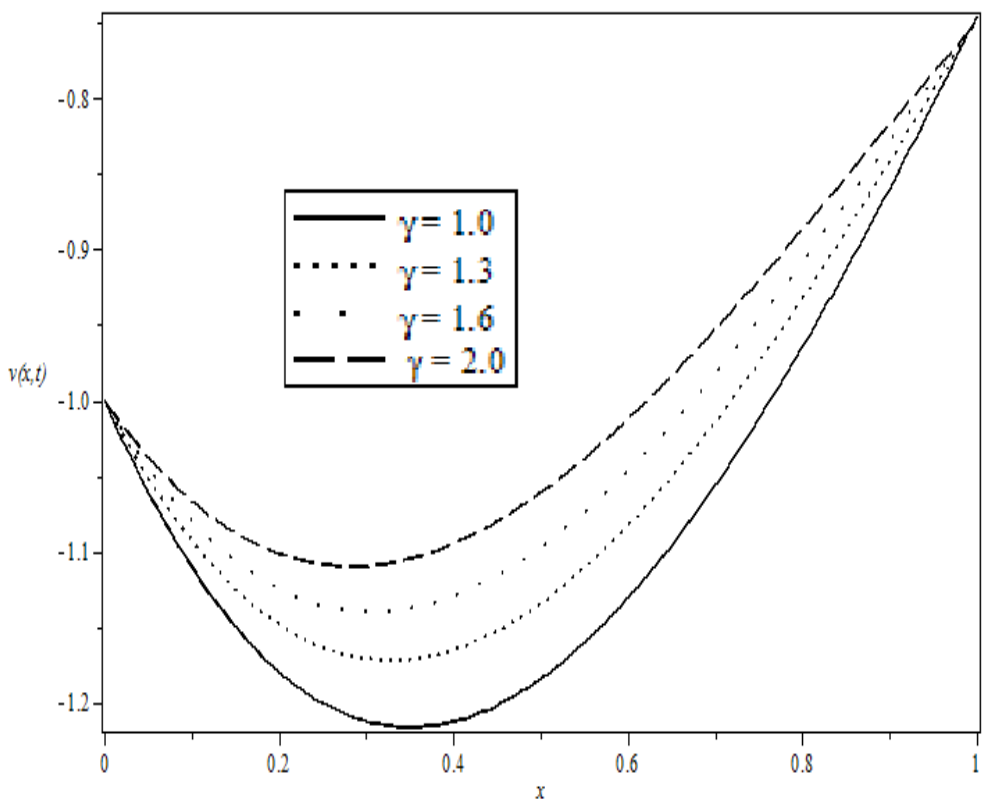

FIGURE 8. Effect of fourth-order parameter $\gamma$ on drainage velocity profile when $\omega=5, \alpha$ $=0.2, \beta=0.4, \beta_{1}=0.7, S_{t}=0.2, \gamma_{1}=0.2, t=3, M=0.5$.

parameter increases the velocity profile in lift problem but in drainage problem, it is clear that the boundary layer thickness is reciprocal to the transverse magnetic field and velocity decreases as flow progresses towards the surface of the fluid are Figures 11 and 12. 


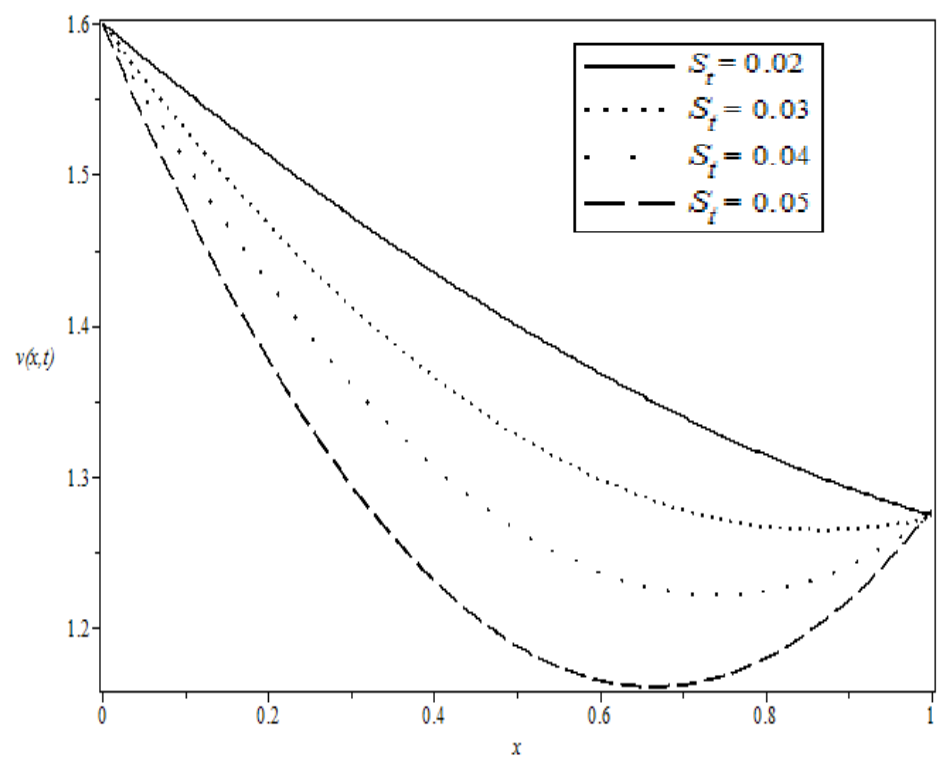

FIGURE 9. Influence of stock number $S_{t}$ on lift velocity profile when $\omega=5, \alpha=0.2, \beta=0.4$, $\beta_{1}=0.3, \gamma=2.0, \gamma_{1}=0.3, t=3, M=0.5$.

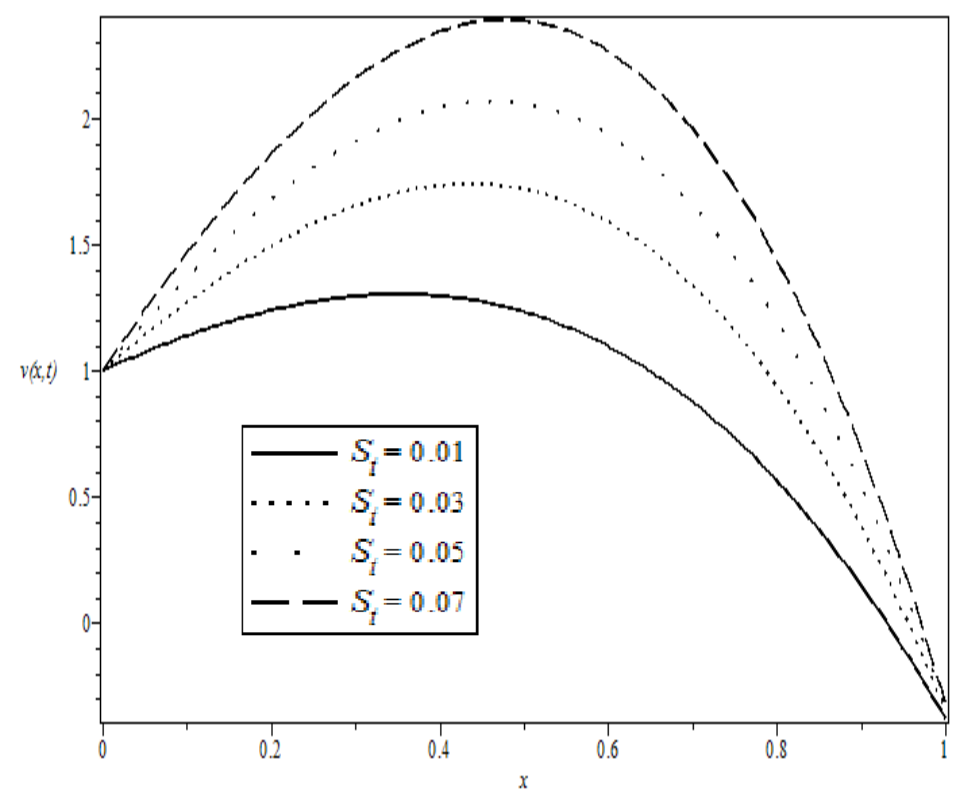

FIGURE 10. Influence of stock number $S_{t}$ on drainage velocity profile when $\omega=5, \alpha=0.2$, $\beta=0.4, \beta_{1}=0.3, \gamma=0.5, \gamma_{1}=0.3, t=5, M=0.5$. 


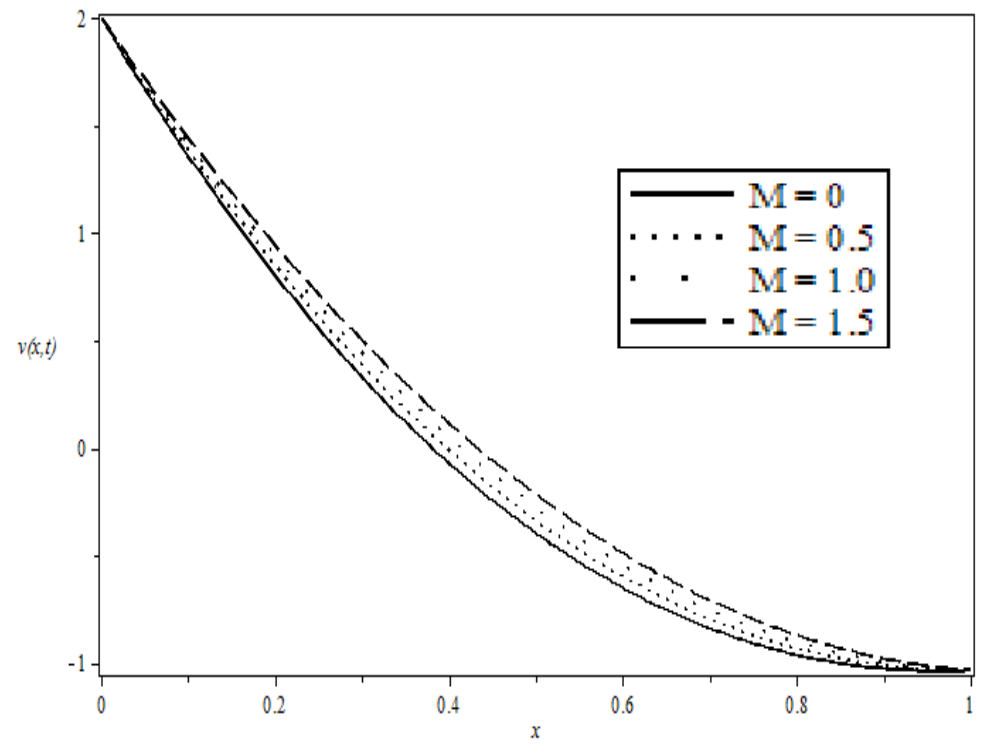

FIGURE 11. Effect of magnetic parameter $M$ on lift velocity profile when $\omega=5, \alpha=0.2, \beta$ $=0.2, \beta_{1}=0.3, \gamma=2.0, \gamma_{1}=0.3, t=3, S_{t}=0.2$.

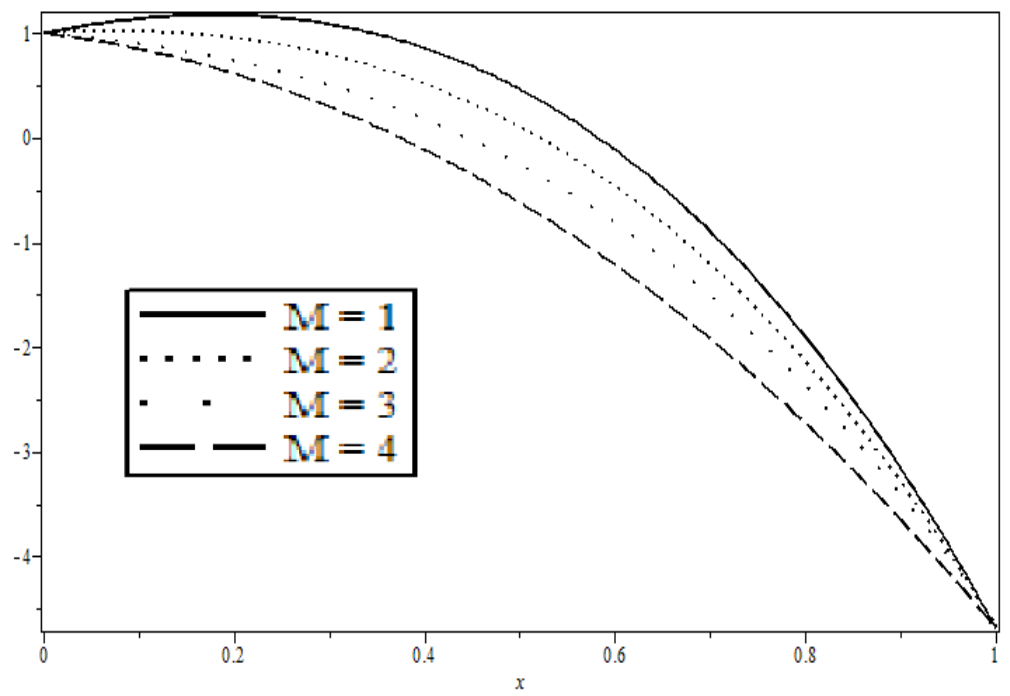

FIGURE 12. Effect of magnetic parameter $M$ on drainage velocity profile when $\omega=5, \alpha=$ $0.2, \beta=0.4, \beta_{1}=0.3, \gamma=2.0, \gamma_{1}=0.3, t=3, S_{t}=0.2$.

\section{Conclusion}

The analytical solutions for unsteady MHD thin layer flow of fourth-order fluid through an oscillating and moving vertical belt is obtained. The belt is translating and oscillating for lift velocity distribution while the belt is only oscillating for drainage velocity profile. The 
non-linear partial differential equations are modeled for both drainage and lift problems and solved by using analytical technique ADM. The graphical results of velocity profile and the effects of physical parameters have been provided and discussed.

\section{References}

1. Gul T, Ghani F, Islam S, Shah RA, Khan I, Saleem N, Sharidan S. Unsteady thin film flow of a fourth grade fluid over a vertical moving and oscillating belt. Journal of Propulsion and Power Research. 2016; 5(3), 223-235.

2. Gul T, Khalid A, Islam S, Shah RA, Khan I, Saleem N, Sharidan S. Heat transfer analysis of MHD thin film flow of an unsteady second grade fluid past a vertical oscillating belt. Journal of PLoS-One. 2014; 9(11), 1-21.

3. Gul T, Ghani F, Islam S, Shah RA, Khan I, Saleem N, Sharidan S, Khan MA. Unsteady MHD thin film flow of a third grade fluid over an oscillating inclined belt embedded in a porous medium. Journal of Thermal Science. 2015; 21, 875-887.

4. Khan NA, Umair BS, Faqiha S, Saifullah, Rehman A. Study of velocity and temperature distributions in boundary layer flow of fourth grade fluid over an exponential stretching sheet. Journal of AIP Advance. 2018; 8, 025011.

5. Hydrodynamic stability and heat and mass transfer flow analysis of MHD radiative fourthgrade fluid through porous plate with chemical reaction. https://www.sciencedirect.com/ science/article/pii/S1018364718317026. Date accessed: 02/01/2019.

6. Akinshilo AT. Steady flow and heat transfer analysis of third grade fluid with porous medium and heat generation. Journal of Science Direct. 2017; 20, 1602-1609.

7. Hayat T, Kara HA, Momoniat E. The unsteady flow of a fourth-grade fluid past a porous plate. Journal of Mathematical and Computer Modeling. 2005; 41, 1347-1353.

8. Gul T, Shah RA, Islam S, Arif M. MHD thin film flows of a third grade fluid on a vertical belt with slip boundary conditions. Journal of Applied Mathematics. 2013; 2013, 1-14.

9. Gul T, Islam S, Shah RA, Khan I, Shafie S. Thin film flow in MHD third grade fluid on a vertical belt with temperature dependent viscosity. Journal of PLoS-One. 2014; 9(6), 1-12.

10. Aiyesimi YM, Okadeya GT, Lawal OW. Unsteady magneto-hydrodynamic (MHD) thin film flow of third grade fluid with heat transfer and no slip boundary condition down an inclined plane. International Journal of Physical Sciences. 2013; 8(19), 946-955.

11. Siddiqui AM, Mahmood R, Ghori QK. Thin film flow of a third grade fluid on a moving belt by He's homotopy perturbation method. International Journal of Nonlinear Science and Numerical Simulation. 2006; 7(1), 7-14.

12. Zaman H, Abbas T, Sohail A, Ali Z. Couette flow problem for an unsteady MHD fourth grade fluid with hall currents. Journal of Applied Mathematics and Physics. 2014; 2, 1-10. 